\title{
Electron spin resonance signal of human nails: increase after irradiation
}

\author{
Seiko Hirota ${ }^{1} \cdot$ Chryzel A. B. Gonzales $^{1} \cdot$ Hiroshi Yasuda $^{1} \cdot$ Ichiro Yamaguchi $^{2}$ - Shin Toyoda ${ }^{3}$
}

Received: 20 September 2020 / Accepted: 3 December 2020 / Published online: 5 January 2021

(c) The Author(s) 2021

\begin{abstract}
We report the phenomenon whereby the electron spin resonance signal of human nails intensify after irradiation. Forty nail samples from three donors were divided into three groups (X-ray irradiated, $\gamma$-ray irradiated, and control). The extent of signal intensified after irradiation was proportional to the given dose under $11 \%$ humidity at $20{ }^{\circ} \mathrm{C}$. This result seemed to contradict previous studies, reporting signal fading. Based on literature and our experimental results, we constructed a model taking both the decay and increase into consideration. Our investigation revealed that rapid deterioration in the signal intensity obscured the increase at humidity levels exceeding $40 \%$.
\end{abstract}

Keywords Electron spin resonance $\cdot$ Nail $\cdot$ Retrospective dosimetry

\section{Introduction}

Electron spin resonance (ESR) has been widely used for basic research and various applications because it can be used to non-destructively detect unpaired electrons in molecules and radicals that exist in samples. Retrospective dosimetry in radiation disasters and accidents is a typical application; for example, atomic bombs [1,2], accidents involving nuclear reactors such as the Chernobyl disaster [3], accidents at nuclear test sites [4], and medical accidents [5].

In the context of retrospective dosimetry, the signal characteristics of many biomaterials, such as teeth, bones, nails, belongings, and everything on-site, have been investigated to find adequate samples that would enable the exposed dose of victims and the environment to be reconstructed $[6,7]$.

A well-researched sample is tooth enamel, for which dose reconstruction methods have been established at doses of $500 \mathrm{mGy}$ and above. However, the current method, which

Seiko Hirota

hirota-seiko@hiroshima-u.ac.jp

1 Research Institute for Radiation Biology and Medicine, Hiroshima University, Kasumi 1-2-3, Minami-ku, Hiroshima 734-0037, Japan

2 National Institute of Public Health, 2-3-6 Minami, Wako-shi, Saitama 351-0197, Japan

3 Okayama University of Science, 1-1 Ridaicho, Kita-ku, Okayama-shi 700-0005, Japan requires a sample to be harvested from a living person, is extremely invasive.

In terms of sample collection, nails are ideal because of the low invasiveness and ease of use of this method. However, previous studies suggested that radiation-induced signals (RISs) decay quickly [8-10] as a result of moisture. To stabilize the RIS, storage in a vacuum desiccator or freezer at less than $-20{ }^{\circ} \mathrm{C}$ was suggested $[11,12]$. The attention of researchers has since shifted to other important issues, especially the separation of signals from noise because mechanical stress also generates a mechanically induced signal (MIS), and the background signal (BKS) exists natively $[8,13,14]$. These signals would have to be removed to enable the radiation dose to be reconstructed from the RIS. The behavior of the RIS under ambient conditions with less humidity has not been studied adequately owing to the shift of researchers' attention to the separation and stabilization of the RIS.

In an ideal laboratory situation, samples can be stored in vacuum desiccators or freezers at less than $-20{ }^{\circ} \mathrm{C}$. However, in on-site accidents or during transportation, maintaining ideal conditions can be difficult because of the severe circumstances outside. To reduce the humidity, nail samples can be stored with silica gel in an airtight container. During our investigations of the signal behavior under these conditions, we observed that the RIS has an increasing component despite its well-known tendency to fade. 
In this work, we validate the existence of the increasing component in the RIS. In this paper, we describe the experimental details and the proposed model.

\section{Theory}

An ESR signal results from the absorption of microwaves by the sample accompanied by the energy transition of the splitting spin states of unpaired electrons under the Zeeman effect. The ESR spectrum is the first derivative waveform of the absorption, and the signal size is defined as the peak-topeak amplitude of the spectrum normalized by the sample weight, as shown in Fig. 1. Radiation-exposed human nail samples contain unpaired electrons derived from radicals generated by radiation and mechanical stimuli.

To verify the existence of an increasing component in the RIS, an attempt was made to observe the linear relationship between the increments and the given dose or the initial intensity of the RIS that correlates with a given dose. In the latter relationship, the impact of the fluctuation in the sensitivity of an individual sample to radiation can be removed. To distinguish only the increment resulting from the RIS, we also monitored the signal behavior of non-irradiated samples as controls.

Resolving the conflict with previous knowledge necessitated us to establish a model that would explain both our data and existing data comprehensively. Previous studies that reported RIS fading were reviewed, and the humidity conditions and decay information were obtained from them.

Based on the literature review, we constructed a model that includes fading as a function of humidity as well as the newly discovered phenomenon of increased signal intensity.

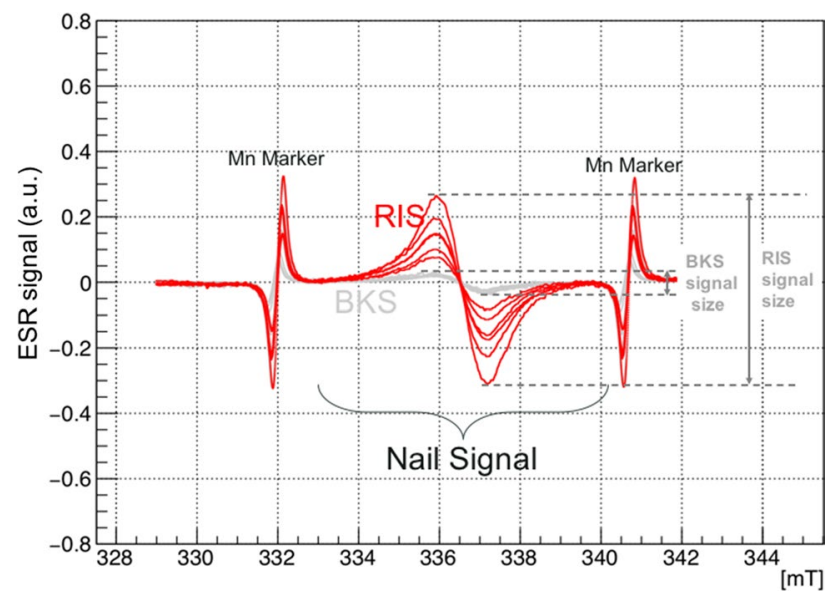

Fig. 1 Typical ESR spectra from nail samples: the red lines represent signals from irradiated samples, and the gray lines represent those from non-irradiated samples after pretreatment (see the "Materials and methods" section for details of the pretreatment)
This model was fitted to the present data, and the relationship between the size of the RIS increment and the initial RIS was estimated.

\section{Materials and methods}

Nail clippers were used to collect 40 nail samples from the fingers and toes of three donors who were in their thirties, forties, and fifties after obtaining their consent. These samples were separated into three groups: X-ray irradiation, $\gamma$-ray irradiation, and control. First, all signals were erased by soaking the samples in water for $12 \mathrm{~h}$ and drying them for four days. After drying, the background signals were monitored for a few days, following which the samples were divided into two groups and irradiated accordingly. The energy of the X-rays was less than $160 \mathrm{keV}$, with a typical energy of $60 \mathrm{keV}$. The $\gamma$-ray source was cesium-137. All samples were stored at $20{ }^{\circ} \mathrm{C}$ and $11 \%$ humidity and monitored for more than one month.

The ESR measurements were conducted on a JES-FA100 instrument (JEOL Ltd., Japan) with an X-band microwave generator $(9.4 \mathrm{GHz})$. The measurement settings were as follows: microwave power, $1 \mathrm{~mW}$; scanning field, 328.5 - 343.5 $\mathrm{mT}$; time constant, $0.3 \mathrm{~s}$; sweep time $30 \mathrm{~s}$; and modulation width, $1 \mathrm{mT}$. The ESR signal intensity was defined as the peak-to-peak amplitude of the averaging spectrum with samples rotated horizontally every $45^{\circ}$ after normalization by sample weight. An uncertainty of $4 \%$ was applied because the main component of the measurement error was expected to be the uncertainty in the vertical position of the sample, and a $1-\mathrm{mm}$ vertical shift of the sample resulted in a $4 \%$ change.

Twelve samples were subjected to X-ray irradiation using a commercial device (Cabinet X-ray system model CP-100, Fraxitron Ltd., USA). In this study, the tube voltage and current were $160 \mathrm{kV}$ and $6.3 \mathrm{~mA}$, respectively. No filter was applied. The most frequent energy of the photons was approximately $60 \mathrm{keV}$. The dose rate was $1.2 \pm 0.04 \mathrm{~Gy} / \mathrm{min}$. Fractionated irradiation was performed to check the linear response, and the total doses of the 12 samples were 23, 47, and $92 \mathrm{~Gy}$ for each group of four samples, respectively.

A Gammacell 40 Exactor Low Dose Rate Research Irradiator (Best Theratronics Ltd., Ottawa, Canada) was used to irradiate the 12 samples with gamma rays. In this irradiator, the sample canister $(10 \mathrm{~cm}$ high and $31.2 \mathrm{~cm}$ in diameter) was located between two cesium-137 sources. The dose rate was $0.79 \mathrm{~Gy} / \mathrm{min}$. The total doses the samples received were 24,48 , and $97 \mathrm{~Gy}$ for each group of four samples, respectively.

To measure the air-absorbed dose in the radiation fields where the samples were located, we used glass dosimeters 
(Dose Ace GD-352 M) for X-rays and GD-302 M (Chiyoda Technol Corporation, Tokyo, Japan) for gamma rays.

During the monitoring period, all samples were stored in airtight plastic bags with silica gel (Medium Granular, Blue, FUJIFILM Wako Pure Chemical Corporation, Japan) to maintain the low humidity level. These plastic bags were kept in the dark box at $20^{\circ} \mathrm{C}$. The humidity and temperature were monitored throughout the monitoring period using a logger (LR5001, Hioki, Japan).

\section{Analysis of Signal Changes}

We conducted a literature search and found three reports that describe RIS fading, as detailed in Table 1. Two of these three reports reported the humidity among the experimental conditions.

In one literature [8], when the samples were stored in a vacuum oven, it was not possible to observe the decay for five days. However, under $60 \%$ humidity, a decline to the background level was observed within five days. Soaking the samples in water, the half-life of RIS was approximately $5 \mathrm{~min}$. The fading in water was reproduced in another study [10].

Based on the first study [8] listed in the table, the fading upon soaking in water follows exponential decay, as shown in Eq. (1).

$I(t)=A e^{-\frac{t}{t_{\text {half }}}}+I_{\text {const }}$

where $I(t)$ is the signal intensity at time $t$, which is the number of days after irradiation. The fading was characterized by three factors: the ratio of decay component $A$, the ratio of constant component $I_{\text {const }}$, and the half-life in units of days $t_{\text {half }}$. The summation of the first and second factors was almost one.

The peak-to-peak amplitude of the spectra was measured after $1 \mathrm{~h}, 1$ day, and 5 days at $60 \%$ humidity and under vacuum [8]. This information was substituted into Eq. (1) to calculate the factors at $60 \%$ humidity and under vacuum. The results are presented in Table 2 to. In our experiment, the humidity was maintained at approximately $11 \%$ for the entire storage period. The three factors of Eq. (1) at $11 \%$ humidity were estimated from values in Table 2 by determining the tendency using the polynomial of humidity. The humidity in the vacuum oven was assumed to be $0 \%$, and that in the water was $100 \%$. As a result, $A$ was $0.29, I_{\text {const }}$ was 0.75 , and $t_{\text {half }}$ was 0.89 .

To consider the phenomenon of increasing signal intensity, a typical saturation curve was used. In addition, based on the analysis of the non-irradiated samples, the term for BKS behavior was added. The model for the experimental data is shown in Eq. (2).

$I(t)=I_{0}+A_{B K S} t+\left(I_{R I S}+a_{R I S} \frac{\left(t-t_{i r}\right)}{\sqrt{1+\left(t-t_{i r}\right)^{2}}}\right)\left(A e^{-\frac{t}{t_{\text {half }}}}+I_{\text {const }}\right)$

where $I_{0}$ is the initial BKS size, $A_{B K S}$ is the increase or decrease in the rate of the BKS, $I_{R I S}$ is the initial RIS, $a_{R I S}$ is the final increment of the RIS, and $t_{i r}$ is the time of irradiation. Moreover, $I_{0}+A_{B K S} t$ expresses the BKS at time $t$, $a_{R I S}\left(t-t_{i r}\right) / \sqrt{ }\left(1+\left(t-t_{i r}\right)^{2}\right)$ describes the increasing behavior of the RIS, and $A \exp \left(-t / t_{\text {half }}\right)+I_{\text {const }}$ is the decay factor for the RIS.

Equation (2) was fitted to the data with the three decay parameters for $11 \%$ humidity.

\section{Results and discussion}

The BKS intensity immediately after pretreatment for eliminating signals before this experiment was $0.061 / \mathrm{mg}$ on average, and its standard error was $0.005 / \mathrm{mg}$ for all samples. The signal intensity is shown in arbitrary units. The breakdown was as follows: from the control group: $(0.052 \pm 0.005) / \mathrm{mg}$; from the group irradiated by X-rays: $(0.089 \pm 0.009) / \mathrm{mg}$, and

Table 2 the thinks of list

\begin{tabular}{llll}
\hline Humidity & $A$ & $I_{\text {const }}$ & $t_{\text {half }}$ \\
\hline Vacuum oven $(0 \%)$ & 0.13 & 0.88 & 0.90 \\
$60 \%$ & 0.82 & 0.22 & 0.65 \\
Water $(100 \%)$ & 0.96 & 0.04 & 0.0047 \\
\hline
\end{tabular}

Table 1 Previous studies that reported RIS fading

\begin{tabular}{llll}
\hline Humidity & Temp. & Decay time & Reference \\
\hline Vacuum oven & $20{ }^{\circ} \mathrm{C}$ & No decay & Sholom et al., 2016 [8] \\
$60 \%$ & $20{ }^{\circ} \mathrm{C}$ & Decay to BKG level in 5 days & \\
Water $(100 \%)$ & $20{ }^{\circ} \mathrm{C}$ & Half-life: $4.7 \mathrm{~min}$ & \\
- & $20{ }^{\circ} \mathrm{C} \sim 40{ }^{\circ} \mathrm{C}$ & Half-life: $300 \mathrm{~h}$ & Symons et al., 1995 [9] \\
- & $25{ }^{\circ} \mathrm{C}$ & Decays completely in 100 days & \\
Water $(100 \%)$ & $20-22{ }^{\circ} \mathrm{C}$ & Half-life: $5 \sim 8$ min & Sholom et al., 2017 [10] \\
\hline
\end{tabular}


from the group irradiated by gamma rays: $(0.054 \pm 0.003) /$ mg.

The intensity of the BKS was measured before irradiation. It was possible to observe a slight increase in BKS: the rate of increase was $(0.0008 \pm 0.0002) / \mathrm{mg} /$ day, $(0.0017 \pm 0.0003) / \mathrm{mg} /$ day, and $(0.0019 \pm 0.0004) / \mathrm{mg} /$ day for the control samples and those irradiated by X-rays and gamma rays, respectively. The ratios of increase relative to the initial BKS intensity were $1.5 \% /$ day, $1.9 \% /$ day, and $3.7 \% /$ day for the control, X-ray, and gamma-ray groups, respectively.

The sensitivities for X-rays and gamma rays were similar. Additionally, the inter-donor variety of sensitivity was not found. The average sensitivity from all samples was $(0.0046 \pm 0.0001) / \mathrm{Gy}$ and $(0.0046 \pm 0.0002) / \mathrm{Gy}$ for X-rays and $(0.0046 \pm 0.0002) / G y$ for gamma rays. According to the Kruskal-Wallis test, the null hypothesis that all data from different donors belong to the same mother distribution was not denied.

Figure 2 shows the typical behavior of the signal intensity from a sample irradiated by X-rays, which the signal additionally increased for the first few days, after which it maintained to increase derived from the BKS.

The $a_{R I S}$ obtained by fitting Eq. (2) to the data after irradiation denotes the maximum size of the additional increment without fading. The value of $a_{R I S}$ was correlated to the given dose for both the X-ray and gamma-ray groups.

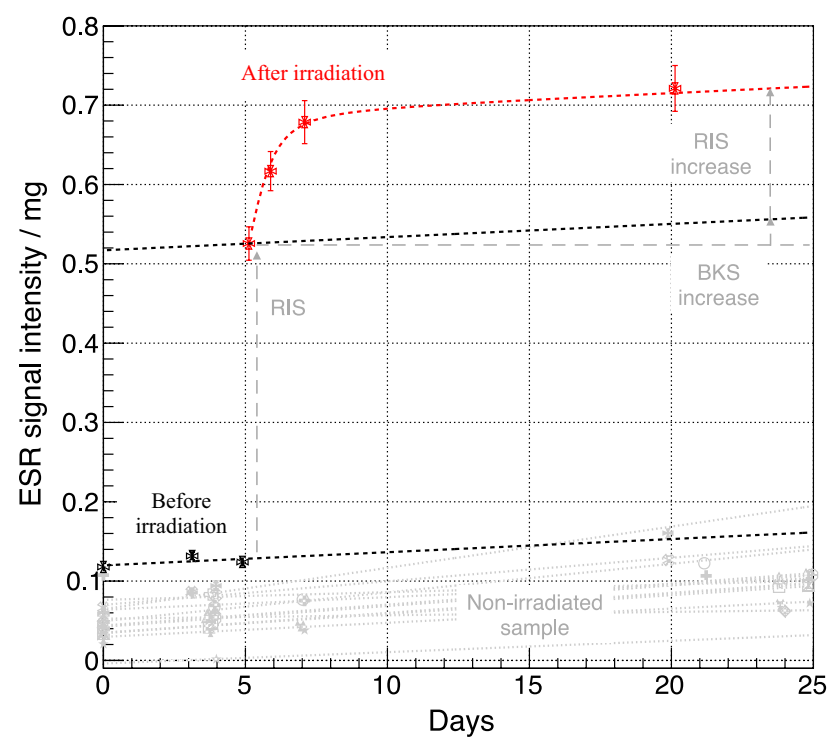

Fig. 2 Typical behavior of signal intensity. This sample was a fingernail from a male donor in his thirties and was irradiated by $92-\mathrm{Gy}$ $\mathrm{X}$-rays. The black points represent the data before irradiation, and the red points show those after irradiation. The gray lines represent the results of monitoring the control samples. The fluctuation in the error bars is the result of sample positioning during ESR measurements. The size of the fluctuation was $4 \%$ for a $1-\mathrm{mm}$ shift of the sample position
The tendency was more explicit in the X-ray group than the gammma-ray group, with a correlation coefficient of 0.94 . For the gamma-ray group, it was 0.38 . When both of these sets of data were merged, the correlation coefficient was 0.63 . The average $a_{R I S} /$ Gy for all samples was 0.0036 , which was $78 \%$ of the average sensitivity.

Figure 3 shows the relationship between $a_{R I S}$ and the initial RIS intensity $I_{R I S}$. This figure implies that the excess from the initial RIS generated by X-rays would have been $107 \%$ if fading did not occur. In reality, the convolution of both the increasing and fading intensity determines the maximum size of the increment. Taking this into account, the full excess of the RIS was approximately $50 \%$ of the initial RIS at $11 \%$ humidity. Some data of the gamma-ray group were mostly consistent with those of the X-ray group. The reason for specific data not being correlated with the initial RIS remains unknown, but it is worthwhile mentioning that these noncorrelated data were collected from one donor-a male in his fifties.

The substitution of the $a_{\text {RIS }}$ values at irradiation of $50 \mathrm{~Gy}$ into Eq. (2) made it possible to calculate the time dependence of the signal intensity under various humidity conditions, as shown in Fig. 4.

At humidity levels in excess of $40 \%$, the fading was dominant; therefore, we can suppose that, at these humidity levels, the increase we observed remained undetected in previous studies. However, even if the humidity was less than $20 \%$, the excess resulting from the increase may not have been apparent at a low dose. The inaccuracy of the

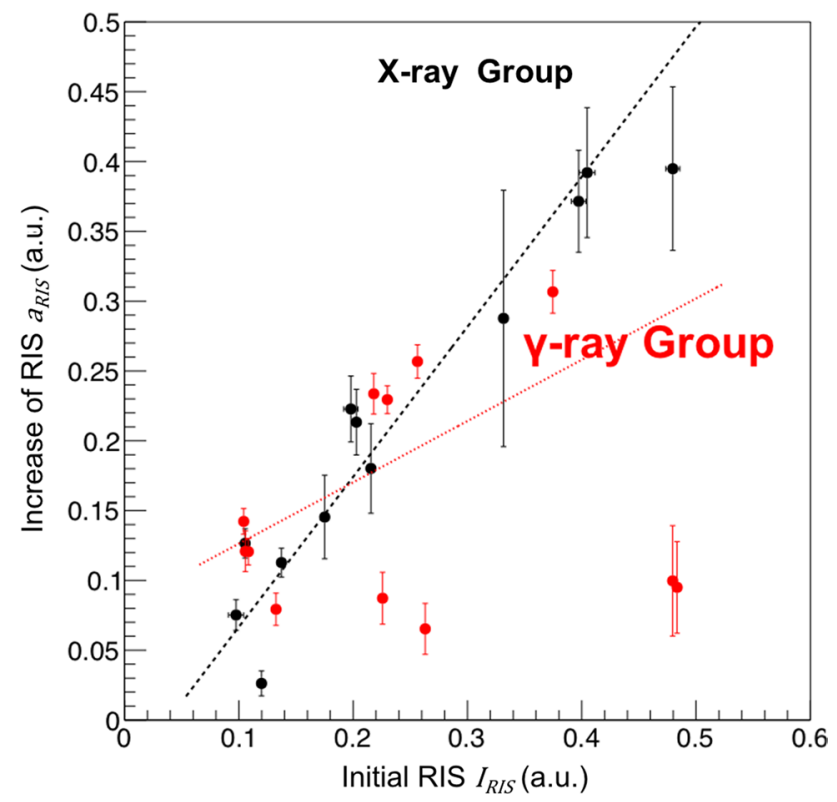

Fig. 3 Relationship between the additional increment $a_{R I S}$ and initial RIS $I_{R I S}$. The black and red dots denote the X-ray and gamma-ray groups, respectively. The error bars were calculated by the error propagation of the sample positioning errors during ESR measurements 


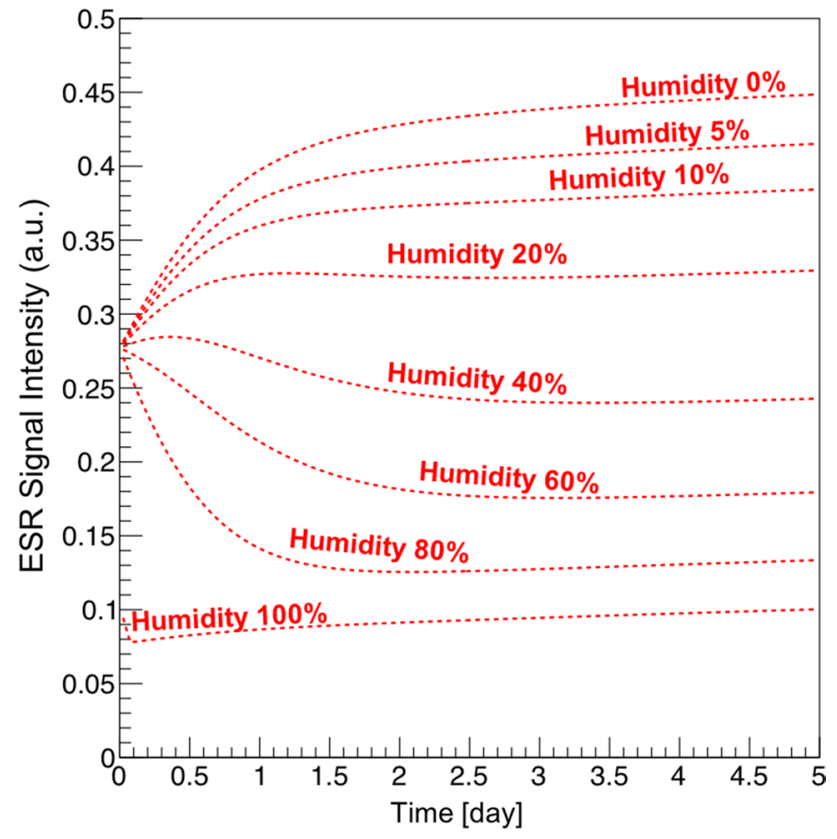

Fig. 4 Calculated intensity for a constant value of $a_{R I S}(50 \mathrm{~Gy})$ at different humidity levels using Eq. (2)

measurement is a few percent of the total signal size of the RIS, MIS, and BKS. This result might explain why the RIS from human nails is well known to undergo fading.

\section{Conclusions}

Under relatively dry conditions, such as humidity levels below $11 \%$, the radiation-induced ESR signal measured from human nails continued increasing for several days after irradiation. The initial response to X-rays and gamma rays was similar for all donors; however, the sample set from one of the donors showed a distinct tendency to differ from others toward the phenomenon of signal increment. Further study is required, including the extent to which the signal intensifies depending on the particular donor.

Acknowledgements This work was supported by the Program of the Network-type Joint Usage/Research Center for Radiation Disaster Medical Science and MEXT/JSPS KAKENHI Grant Number 18KK0147. We would like to thank Editage (www.editage.com) for English language editing.
Funding This experiment was approved by the ethics committee of Hiroshima University (No. E-2843).

Open Access This article is licensed under a Creative Commons Attribution 4.0 International License, which permits use, sharing, adaptation, distribution and reproduction in any medium or format, as long as you give appropriate credit to the original author(s) and the source, provide a link to the Creative Commons licence, and indicate if changes were made. The images or other third party material in this article are included in the article's Creative Commons licence, unless indicated otherwise in a credit line to the material. If material is not included in the article's Creative Commons licence and your intended use is not permitted by statutory regulation or exceeds the permitted use, you will need to obtain permission directly from the copyright holder. To view a copy of this licence, visit http://creativecommons.org/licenses/by/4.0/.

\section{References}

1. Ikeya M (1984) Japanese J Rad Phys. https://doi.org/10.1143/ JJAP.23.L697

2. Hirai Y (2019) Tooth enamel ESR doses and cytogenetic doses of Nagasaki atomic-bomb survivors in comparison with DS02R1 doses. Int J Radiat Biol 95:321-328

3. Ishii H (1990) J Nucl Sci Tech. https://doi.org/10.3327/ jnst.27.1153

4. Zhumadilov Z (2012) IPSHU English Res Rep Ser. https://doi. org/10.15027/33634

5. Trompier F (2010) Health Phys. https://doi.org/10.1097/01. HP.0000346334.78268.31

6. Nakajima T (1982) Int J Appl Radiat Isot. https://doi. org/10.1016/0020-708x(82)90236-8

7. Dalgarno B (1989) Int J Appl Radiat Isot. https://doi. org/10.1016/0883-2889(89)90034-8

8. Sholom S (2016) J Radiat Med. https://doi.org/10.1016/j.radme as.2016.02.014

9. Symons MCR (1995) Radiat Prot Dosim. https://doi.org/10.1093/ oxfordjournals.rpd.a082591

10. Sholom S (2017) Radiat Phys Chem. https://doi.org/10.1016/j. radphyschem.2017.06.009

11. Reyes R (2012) Health Phys. https://doi.org/10.1097/HP.0b013 e31824ac338

12. Reyes R (2008) Radiat Environ Biophys. https://doi.org/10.1007/ s00411-008-0178-8

13. Romanyukha A (2007) Radiat Meas. https://doi.org/10.1016/j. radmeas.2007.05.026

14. Sholom S (2019) Radiat Prot Dosim. https://doi.org/10.1093/rpd/ ncz019/5365232

Publisher's Note Springer Nature remains neutral with regard to jurisdictional claims in published maps and institutional affiliations. 